\title{
BRITTLE AND DUCTILE FRACTURE AT THE ATOMISTIC CRACK TIP IN COPPER CRYSTALS*
}

\author{
Y.W. Zhang, T.C. Wang, Q.H. Tang \\ LNM, Institute of Mechanics, Chinese Academy of Sciences \\ Beijing, China. 100080
}

(Received November 16, 1994)

(Revised February 28, 1995)

\section{Introduction}

The interactions of crack and dislocations or the competitions between dislocation emission and cleavage are perhaps one of the most important factors to determine the failure behaviours of materials. The analyses based on the continuum elasticity have originally been investigated by Rice and Thomson(1) and subsequently extended by $\mathrm{Ohr}(2), \mathrm{Li}(3), \mathrm{Rice}(4)$ and among others. The analyses are unsatisfactory because of their use of the continuum elasticity. Due to the nonlinear and atomic lattice effects, the continuum elasticity is not suitable for describing the deformation behaviours very near the crack tip(Zhang and Wang(5)). So it is essential to analyze the dislocation nucleation and emission from crack tip by atomistic simulation unitizing an appropriate interatomic law.

The molecular dynamics simulations of crack processes were investigated by many researchers. Dienes and Paskin(6) put their emphasis on the crack propagation velocity and some environmental factors on fracture, an assumed triangular lattice was used in their simulation. The brittle and ductile transition in intrinsic fracture behaviour of crystal was studied by Cheung and Yip(7), the thermo-activation effect was included in their analysis. The crack tip deformation in Al using an embedded atom potential was investigated by Hoagland, Daw, Foiles and Baskes $(8)$, three crack/crystal orientations were examined and two of which behave in a pure brittle mode without dislocation emission.

Experimental results show that the brittle cleavage especially in metal is often accompanied by considerable amount of dislocation activation. Cracks often grow in a zigzag manner along the alternative slip plane of the emitted dislocations ( $\mathrm{Ohr}(2)$, Hong and Laird(9)). On the other hand, the dislocation emission and crack propagation depend strongly on the geometric relation between crack and crystal orientation. This effect must be considered so as to properly describe the behaviours of ductile and brittle fracture in crystal.

In the present paper, the molecular dynamics method is used to simulate ductile and brittle failure at the atomistic crack tip in copper with FCC structure. Here we only consider the cases of a crack tip intersected by a single inclined slip plane. The effects of geometry of crack and crystal orientation on the crack tip processes are studied.

\footnotetext{
*The project is supported by the National Natural Science Foundation of China.
} 


\section{Calculation Method}

\section{A. Interatomic Potential}

The interatomic potential used here is the "N-body" potential proposed by Finnis and Sinclair(10) and constructed by Ackland et al(11). The ansatz they used is:

$$
E_{t o t}=-\sum_{i} \rho_{i}^{\frac{1}{2}}+\frac{1}{2} \sum_{i} \sum_{j(i \neq j)} V_{i j}
$$

$\rho$ is the second moment of the density of states, and

$$
\rho_{i}=\sum_{j(i \neq j)} \Phi_{i j}
$$

$V_{i j}$ and $\Phi_{i j}$ are functions only of the interatomic distance, and can be obtained by assuming some function forms and then fitting to the experimental data.

\section{B. Method of Solution}

The border discrete atoms are prescribed by the mode I anisotropic elastic $\mathrm{K}$ displacement field. The loading rate $\mathrm{K}_{\mathrm{I}}$ is used as a loading parameter. In the present simulation, $\mathrm{K}_{\mathrm{I}}=0.0354 M P a \sqrt{\mathrm{m}} / \mathrm{ps}$. The inner atoms follow law of Newton

$$
\mathrm{F}_{i}=-\frac{\partial E_{t o t}}{\partial \mathbf{r}_{i}}=m_{i} \dot{\mathbf{v}}_{i}
$$

The present molecular dynamics calculations are carried out by the Leapfrog Algorithm as follows

$$
\left\{\begin{array}{l}
\mathbf{v}_{i}\left(t+\frac{\Delta t}{2}\right)=(1-\eta) \mathbf{v}_{i}\left(t-\frac{\Delta t}{2}\right)+\frac{F_{i}}{m_{i}} \Delta t \\
\mathbf{r}_{i}(t+\Delta t)=\mathbf{r}_{i}(t)+\mathbf{v}_{i}\left(t+\frac{\Delta t}{2}\right) \Delta t \\
\mathbf{v}_{i}(t+\Delta t)=\frac{1}{2}\left[\mathbf{v}_{i}\left(t+\frac{\Delta t}{2}\right)+\mathbf{v}_{i}\left(t-\frac{\Delta t}{2}\right)\right]
\end{array}\right.
$$

In Eqs.(3)(4), $m_{i}$ is the mass of the $i$-th atom, $\eta$ is the numerical damping parameter which is taken to be 0.1 for relaxation processes and zero for loading processes. The above scheme provides an update formulation from the current time $t$. The time step in present calculation is taken to be $1.18 \times 10^{-14} \mathrm{~s}$. In all simulations, the temperature of the systems is taken to be $0 \mathrm{~K}$.

\section{The Atom Lattice Geometry}

The parallelepiped with a slit is used as the simulated cell in present calculations. The case in which the coordinate system is selected to be $x y$ and $z$ axes along $(110),\langle 111\rangle$ and $\langle 112\rangle$ directions respectively is the one of the inclined angle $\theta=0^{\circ}$ (see Fig.1). In FCC crystal, a full dislocation moves in the $\langle 110\rangle$ direction on $\{111\}$ plane. The periodicity along $(111)$ is 3 layers, along $\langle 110\rangle$ is 2 layers and along $\langle 112\rangle$ is 6 layers.

A full dislocation in copper is generally dissociated into two Shockley partial dislocations, between the two partial dislocations, there is a faulted plane. As for the periodicity condition is used along (112) direction, the nucleation and motion of partial dislocations can be described with present lattice configuration. The atom arrangement projected on the a $\{112\}$ plane is just simple cubic lattice, so the partial dislocation can be clearly observed.

The length of the simulated cells along $x$-direction is $30 a_{0}$ ( $a_{0}$ is the lattice constant), the width along $y$-direction $40 a_{0}$. The distance between the crack tip and the left boundary is $15 a_{0}$. The separation of the 


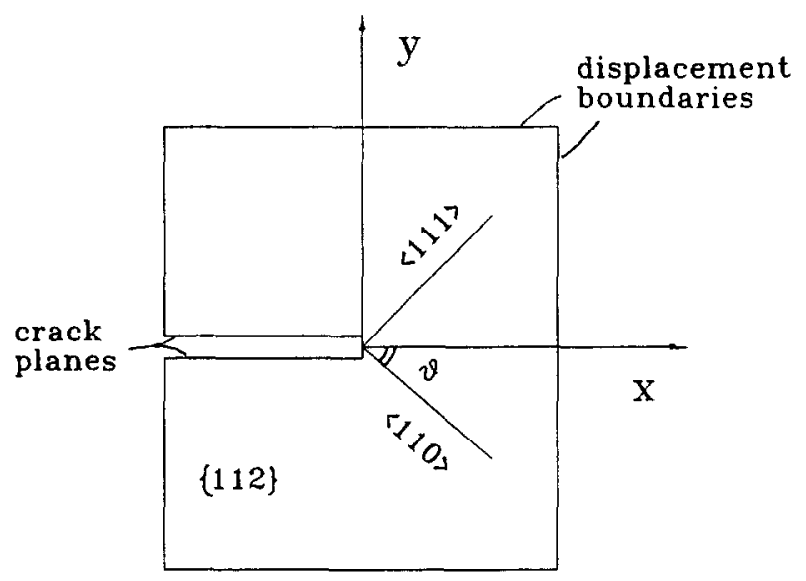

Figure 1. Schematic diagram for the simulated atom model, $\theta$ is the inclined angle of slip plane.

upper and lower crack planes is taken to be $\sqrt{3} a_{0}$ which is larger than $1.22 a_{0}$, the cutoff distance of the potential. The number of atoms used in the present simulations for each case is about $\mathbf{5 7 9 0 .}$

\section{The Boundary Conditions}

The treatments of boundary conditions are of crucial importance to the accuracy of the final results as pointed out by deCelis, Argon and Yip(12). But both force boundary and displacement boundary suffer serious deficiency. For the displacement boundary, there is no way for the dislocations emitted from the crack tip to penetrate the boundary. For the force boundary, although no resistance to the penetration of dislocations is involved, the forces applied to the border atoms will be changed when the dislocations emitted from the crack tip are near the boundary. In the present simulation, the boundary conditions applied to the boundary of the discrete atom region have been that of a prescribed displacement distribution dictated by mode I anisotropic $\mathrm{K}$ field in $\mathrm{x}$-y plane(Sih and Liebowitz(12)). Along z-direction, a six layer periodic representation is applied. Although this displacement boundary is less accurate, it is easy to implement. If the length from the crack tip to the boundary is large enough, the effect of the boundary on the nucleation and emission of dislocations may be neglected. But if the leading dislocation arrives at the boundary, the effect of the displacement boundary can't be neglected.

Here the boundaries are assumed to represent the barriers to the dislocation movement. Thus dynamic pile-ups of the trailing dislocations emitted sequentially from crack tip will be formed. Pile-ups of dislocations will inhibit the emission of dislocations and promote brittle cleavage. Therefor the effect of the displacement boundary will promote the ability of brittle crack extension.

\section{Simulated Results and Discussions}

In present letter, several cases with different inclined angle $\theta$ have been calculated. The following results are obtained.

For $0^{\circ} \leq \theta<16^{\circ}$, although the copper is ductile crystal, the fully brittle cracking is observed in the atomistic crack tip along the inclined slip plane(see Fig. 2 for $\theta=15^{\circ}$ ). The reason is that the resolved shear stress along the inclined slip plane is small, so it is difficult to nucleate a dislocation along this slip plane to blunt the crack. The other slip systems are more difficult to nucleate the dislocations because the intersection of the crack plane and slip plane is not coincident with crack tip front. If no dislocation 


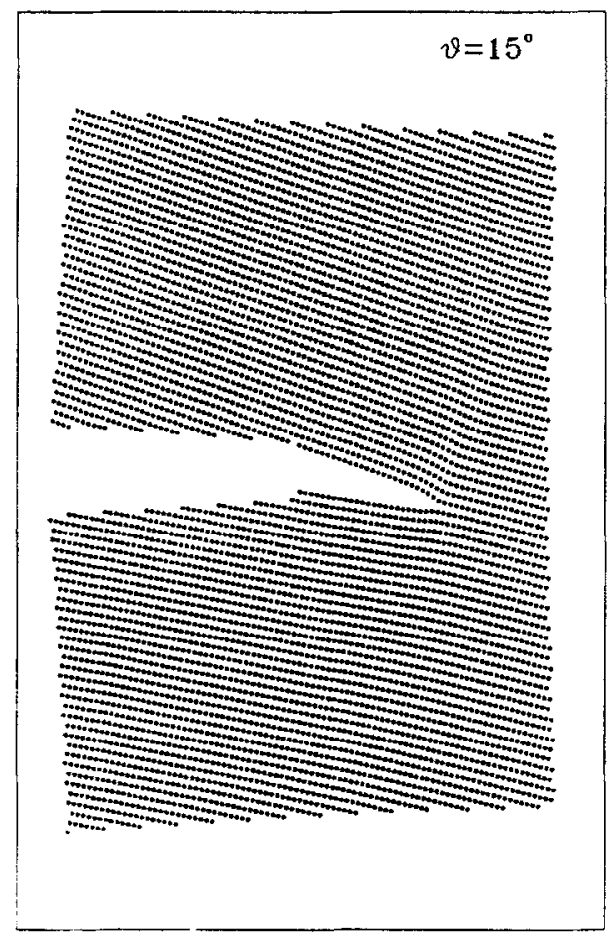

Figure 2. The atom configuration for $\theta=15^{\circ}$ at $K_{I}=1.0 \mathrm{MPa} \sqrt{\mathrm{m}}$. Pure brittle cracking occurs along the slip plane.

is emitted, the crack can't be blunted, the brittle cleavage inevitably occurs. For the crack propagating along the prolongation of crack plane in the anisotropic crystal, the critical stress intensity factor for crack propagation is (Sih and Liebowitz (12))

$$
K_{I c}=\sqrt{2 \gamma_{s}}\left\{\left(\frac{s_{11} s_{22}}{2}\right)\left[\left(\frac{s_{22}}{s_{11}}\right)^{\frac{1}{2}}+\left(\frac{2 s_{12}+s_{66}}{2 s_{11}}\right)\right]\right\}^{-\frac{1}{2}}
$$

where $s_{i j}$ are parameters related with the compliance constants with indices 1,2 and 6 referring to crack propagation, normal of the crack plane and direction of plane strain respectively(Sih and Liebowitz(12)). $\gamma_{s}$ is the surface energy density. For copper, $\gamma=1.79 \mathrm{Jm}^{-2}(\operatorname{Rice}(4))$, so the $K_{I c}=0.52 \mathrm{MPa} \sqrt{\mathrm{m}}$. This is the same as given by $\mathrm{Ohr}$ and $\mathrm{Chang}(13)$, but less than $0.64 \mathrm{MPa} \sqrt{\mathrm{m}}$ given by $\mathrm{Ohr}(2)$ at room temperature. For $\theta=0^{\circ}$, our simulated result is $0.666 \mathrm{MPa} \sqrt{\mathrm{m}}$, which is slightly larger than the above results. This may be the effect of high loading rate of the present simulation.

For $16^{\circ} \leq \theta<25^{\circ}$, although dislocations are emitted, the brittle cracking also occurs along the slip plane before the leading dislocation reaches the displacement boundary (Fig. 3 for $\theta=18^{\circ}$, the dislocation marked by a circle). In the angular range, dislocation emission will shield the crack tip stress and make it more difficult for nucleating the dislocations. This inhibits the crack blunting and the brittle cracking occurs. It seems that the emission of dislocations may not be a sufficient condition for continuing blunting and may not be taken as a criterion for ductile crystal.

For $25^{\circ} \leq \theta<60^{\circ}$, many dislocations are emitted and the crack tip is blunted (Fig.4 for $\theta=45^{\circ}$ ). For copper, a full dislocation always dissociates into two partial dislocations. When the leading partial dislocation is blocked by the displacement boundary, a pileup of dislocations is set up along the slip plane. 


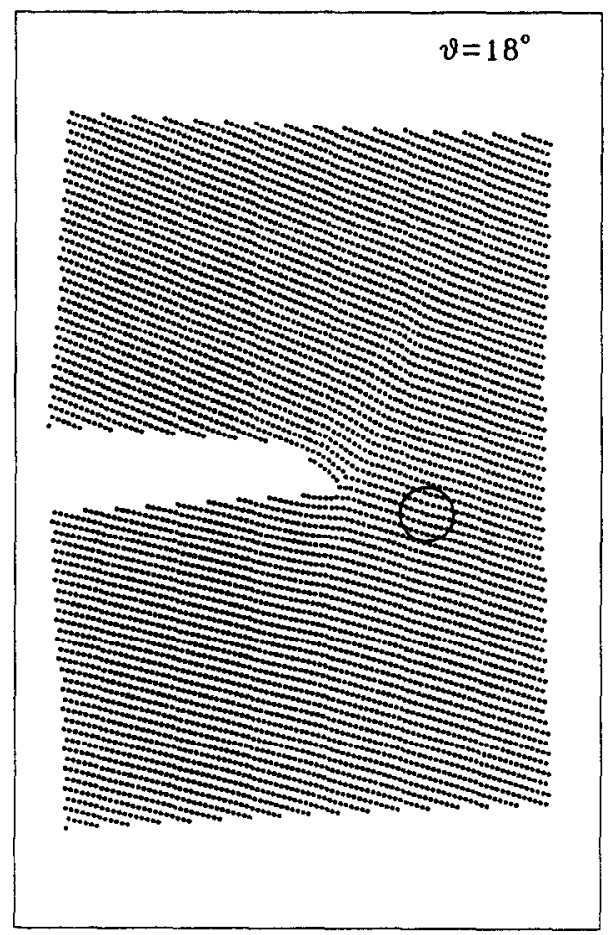

Figure 3. The atom configuration for $\theta=18^{\circ}$ at $K_{l}$ $=0.96 \mathrm{MPa} \sqrt{\mathrm{m}}$. Although a dislocation is emitted, the brittle cracking also occurs along the slip plane before the emitted dislocation reaches the border.

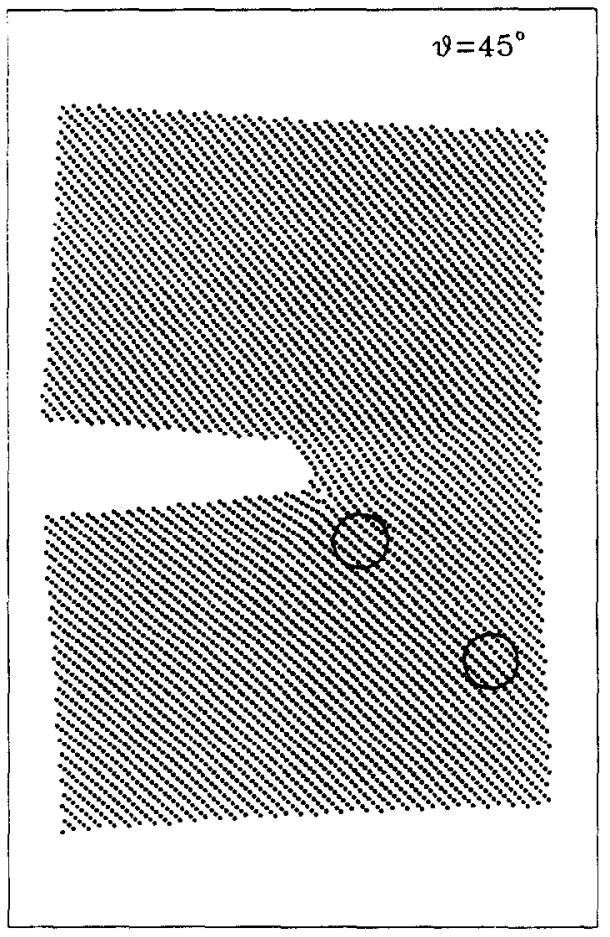

Figure 4. The atom configuration for $\theta=45^{\circ}$ at $K_{I}$ $=1.0 \mathrm{MPa} \sqrt{\mathrm{m}}$. Dislocations are emitted and the first one reaches the border. The crack is blunted by the emitted dislocation.

With continued loading, the cracking occurs along the slip plane of the emitted dislocations (see Fig.5 also for $\theta=45^{\circ}$ ). It seems that if there is no barrier at the slip plane, the crack should be more blunted. So in this angular range, crack tip processes may exhibit a ductile fashion if without the barrier. The existence of the barrier to dislocation motion has strong effect on dislocation emission and cleavage at the crack tip.

For the cases of $60^{\circ} \leq \theta \leq 90^{\circ}$, also many dislocations are emitted along the slip plane and pileups are also observed at the borders. But in this angular range, serious blunting occurs at crack tip and no cleavage along the slip plane is observed. The activation and emission of the secondary slip systems are also observed (see Fig.6 for $\theta=70^{\circ}$ and Fig.7 for $\theta=90^{\circ}$ ). From Fig.6, it can be seen that a pair of partial dislocations combine to form a full dislocation. After the formation of the full dislocation at a pile-up end, it becomes unstable with increasing the loading and results in a dislocation reaction which may be that a full dislocation is decomposed into a Frank partial and a Shockley partial(see dislocations marked by double circles). The Frank partial is a sessile dislocation which remains at the pile-up end. The Shockley partial is glissile dislocation which moves away from the pile-up end. This energetically unfavorable process can take place only at large external loading and partly relaxes the stress concentration at the pile-up end. The dislocation emissions in the secondary slip system are also observed in this case. From Fig.7, it can be seen that the crack tip exhibits a super-blunting manner. With increasing the loading stress intensity factor, a very complicate processes take place at the crack tip. The twinning and the void nucleation can be clearly seen ahead of the crack tip and the secondary slip systems are activated. Some dislocations not emitted from the crack tip are also observed. The dislocation reaction same as Fig. 6 also occurs. 


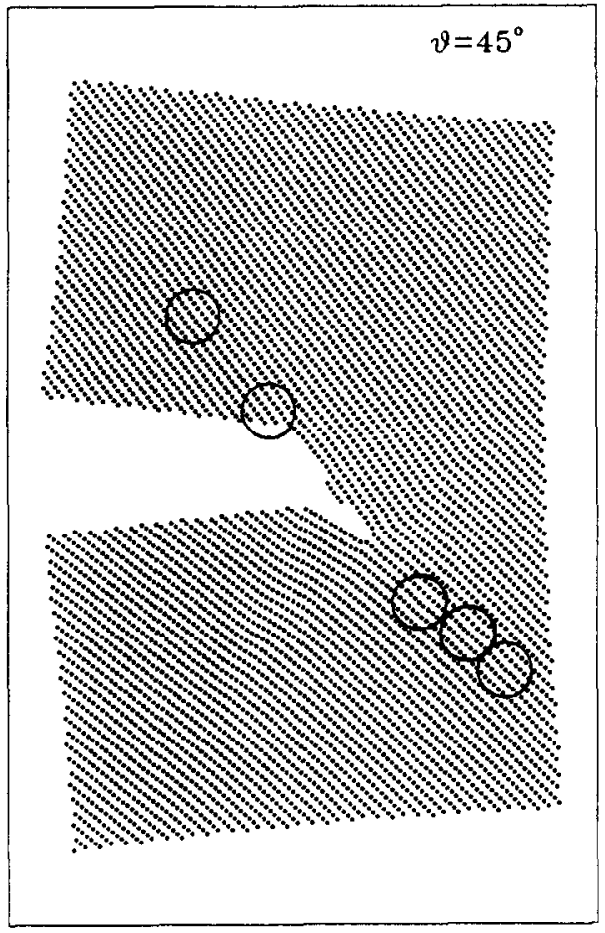

Figure 5. The atom configuration for $\theta=45^{\circ}$ at $K_{I}=1.6 \mathrm{MPa} \sqrt{\mathrm{m}}$. Because of the blocking of the first dislocation, continuing emitting dislocation becomes difficult, the brittle cracking occurs along the slip plane.

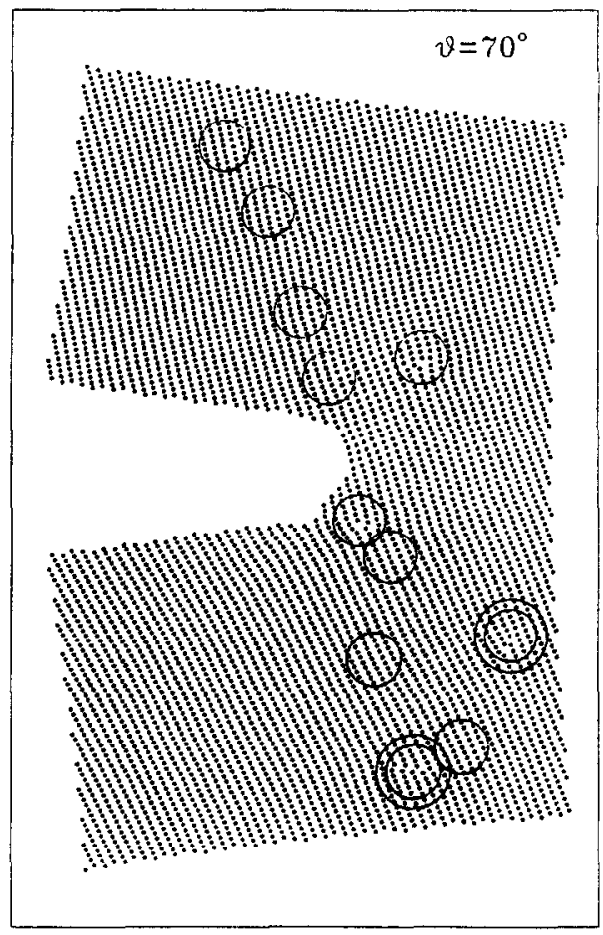

Figure 6. The atom configuration for $\theta=70^{\circ}$ at $K_{I}=2.0 \mathrm{MPa} \sqrt{m}$. The crack tip process exhibits a ductile fashion. A dislocation reaction is also observed.

The variation of the critical stress intensity factors for brittle cracking and for dislocation emission from crack tip with inclined angle $\theta$ obtained by our molecular dynamics simulations are shown in Fig.8. For $0^{\circ} \leq \theta<16^{\circ}$, the brittle cleavage occurs at the atomistic crack tip before the dislocation emission. For $16^{\circ} \leq \theta \leq 90^{\circ}$, the dislocation emission occurs before the cleavage. The inclined angle for the minimum critical stress intensity factor for dislocation nucleation is approximate at $70^{\circ}$.

According to the Rice(4), the dislocation nucleation criterion along an inclined plane under pure mode I loading can be written as

$$
K_{l e}=\frac{\sqrt{\gamma_{u s} p(\theta)}}{f(\theta)}
$$

where

$$
\begin{gathered}
f(\theta)=\cos ^{2}\left(\frac{\theta}{2}\right) \sin \left(\frac{\theta}{2}\right) \\
p(\theta)=\Lambda_{\alpha \alpha}^{(\theta)^{-1}} \quad(\operatorname{sum} \alpha) \\
\Lambda_{\alpha \beta}^{(\theta)}=R_{\alpha \gamma} \Lambda_{\gamma \delta} R_{\beta \delta} \quad(\text { sum } \gamma \text { and } \delta)
\end{gathered}
$$

$\Lambda_{\alpha \beta}$ is the matrix for anisotropic materials (see Wang, Shih and Suo (15)), $\mathbf{R}$ is the rotation matrix, $\gamma_{u s}$ is the unstable stacking energy for dislocation nucleation. Since the $\gamma_{u s}$ depends on material, temperature and loading rate and shows a great uncertainty(Rice (4)), here we take $\gamma_{u s}$ as a parameter to fit our molecular dynamics result at $\theta=70^{\circ}$. The obtained $\gamma_{u s}$ is equal to $0.438 \mathrm{~J} / \mathrm{m}^{2}$. By using this result, the angular 


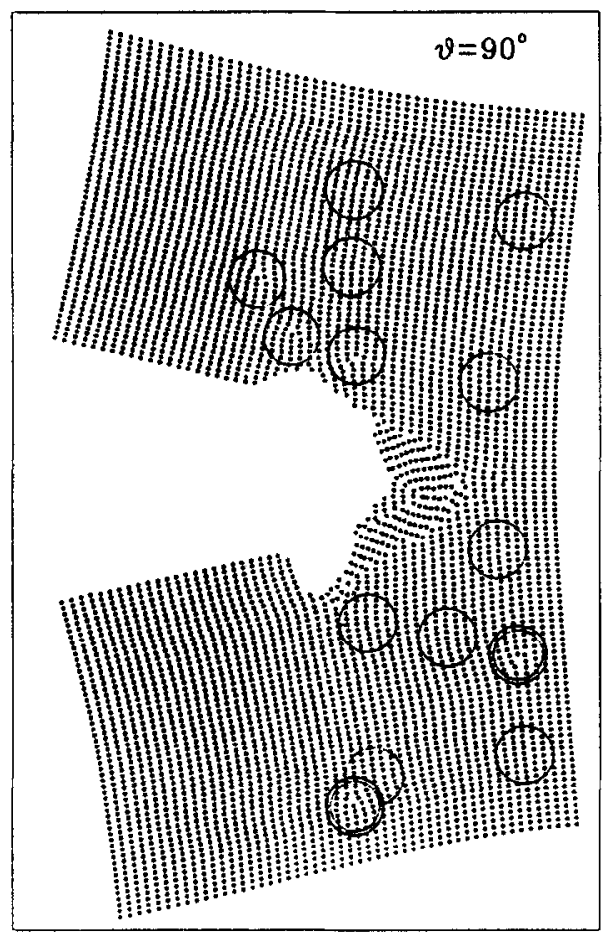

Figure 7. The atom configuration for $\theta=90^{\circ}$ at $K_{I}=$ 3.5MPa $\sqrt{m}$. The superblunting, secondary slip activation, twinning, void nucleation and dislocation reaction are clearly observed.

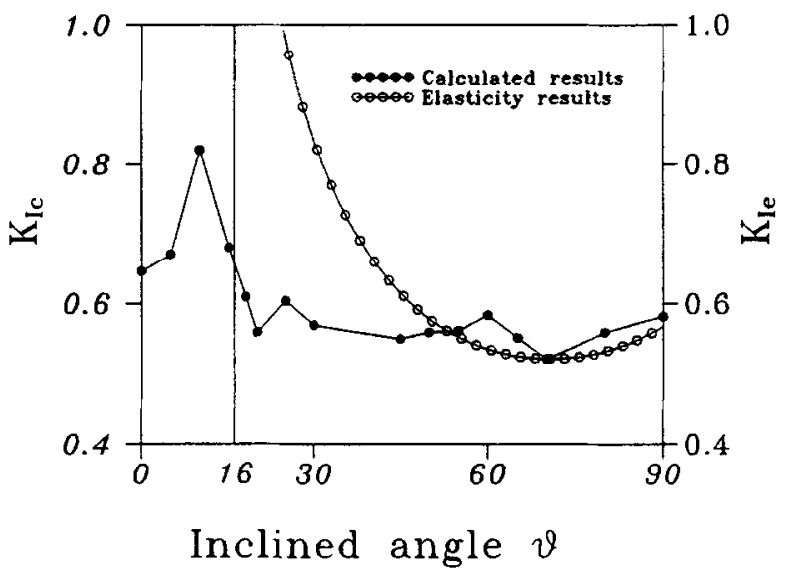

Figure 8. Variations of stress intensity factors for cleavage and dislocation nucleation with the inclined angle $\theta$ for molecular dynamics results and elasticity results.

dependence of dislocation nucleation criterion expressed by Eq.6 is plotted also in Fig.8. It can be seen that although the inclined angle for the minimum stress intensity factor is the same, a great difference exists between the molecular dynamics results and elasticity results. Our simulations show that both cleavage and the dislocation nucleation are sensitive to the atom configuration near crack tip. Our molecular dynamics method can reflect such an atom configuration, but the elasticity theory can't.

\section{$\underline{\text { Summary }}$}

Although our calculations contain some uncertainty associated with the potentials and boundary conditions, they do lead to some physical insights about the crack tip processes. The above analyses show that geometry of the crack and crystal orientations has a strong effect on the crack tip processes. This effect has also been observed by Anderson and Rice(14). The present simulations show that the crack tip may exhibit a pure cleavage, or cleavage following dislocation emissions, or cleavage following the pile-ups of the emitted dislocations at barriers, or blunting or superblunting by the emitted dislocations. If no dislocations are emitted, the crack will propagate in fully brittle manner. If dislocations are emitted from the crack tip, it doesn't mean that crack may continue nucleating dislocations and blunting itself. The pile-ups of emitted dislocations play an important role in the cracking. In the present simulation, the crack propagation is along the slip direction on slip plane, this is in agreement with experimental observations of Ohr(2) and Hong and Laird(9). A typical ductile failure with nucleating a void in front of crack tip is observed, this also agrees with the experimental failure mode of ductile crystal. An edge dislocation reaction is observed, which partly relaxes the stress concentration at the pileup end. 
The shielding effect of dislocation has been paid much attention in previous researches. As for the crack tip processes are very complicate, even the blunting or superblunting phenomenons are observed at certain crack and crystal orientations, the blunting effect of emitted dislocations should be considered in the criterion for the brittle vs. ductile transition at atomistic crack tip.

Our simulations also show that the atom configuration near crack tip may influence the critical conditions for cleavage and dislocation nucleation greatly. The elasticity theory doesn't reflect the atom arrangement near crack tip, so great uncertainty may arise when one studies the dislocation nucleation from crack tip by using elasticity theory.

\section{References}

1. J.R. Rice and R. Thomson, Philos. Mag. 29, 73 (1974).

2. S.M. Ohr, Materials Science and Engineering 72, 1 (1985).

3. J.C.M. Li, Scripta metallurgica 20, 1477 (1986).

4. J.R. Rice, J. Mech. Phys. Solid. 40, 239 (1992).

5. Y.W. Zhang and T.C. Wang, J. App. Phys. (1995) (to be published).

6. G.J. Dienes and A. Paskin, J. Phys. Chem. Solids 48, 1015 (1987).

7. K.S. Cheung and S. Yip, Phys. Rev. Lett. 65, 2804 (1990).

8. R.G. Hoagland, M.S. Daw, S.M. Foiles and M.I. Baskes, J. Mater. Res. 5, 313 (1990).

9. S.I. Hong and C. Laird, Fat. Fract. Eng. Mater. Struct. 14, 143 (1991).

10. M.W. Finnis and J.E. Sinclair, Philos. Mag. 50, 45 (1984).

11. G.J. Ackland, G. Tichy, V. Vitek and M.W. Finnis, Philos. Mag. A56, 735, (1987).

12. G.C. Sih, H. Liebowitz, in Fracture: an advanced treatise Vol.2 p.67 Ed. H. Liebowitz, Academic Press, New York and London.

13. S.M. Ohr and S.J. Chang, J. App. Phys. 535645 (1982)

14. P.M. Anderson and J.R. Rice, Scripta metallurgica 20, 1467 (1986).

15. T.C. Wang, C.F. Shih and Z. Suo, Int. J. Solids Struct. 29, 327 (1992). 